\title{
Estudio de la Pareja Estéril
}

\author{
Fabio Sánchez Escobar * \\ Jairo E. Garcia Gómez * \\ Jesús De los Ríos Osorio ** \\ William Mejía***
}

Relato presentado en el XIII Congreso Colombiano de Obstetricia y Ginecología. Bogotá, diciembre de 1979.

Trabajo ejecutado en el Departamen. to de Obstetricia y Ginecología.

Grupo de Ginecología Endocrina Universidad de Antioquia. Hospital Universitario San Vicente de Paúl - Medellín.

\section{Colaboradores}

Luis Fernando Palacio:

Planeación - U. de A.

William Mejía:

Análisis estadístico - U. de A.

Jaime Bedoya:

Siquiatría - U. de A.

Alberto Abad:

Radioinmunoanálisis - Clínica Soma

Constanza Díaz:

Patología - U. de A.

* Profesor Departamento de Obstetricia y Ginecología - Grupo de Ginecologia Endocrina. Universidad de Antioquia.

** Instructor adhonorem - Departamento de Urología - Universidad de Antioquia y Médico Andrólogo. Profamilia.

*** Jefe del Departamento de Estadistica Escuela Nacional de Salud Pública Universidad de Antioquia.
Aníbal Castañeda: Laparoscopia - Profamilia

Humberto Uribe: Neurocirugía - U. de A.

Guillermo Henao: Ginecología Endocrina - U. de A.

Residentes, internos y estudiantes del Departamento.

\section{INTRODUCCION}

Durante las últimas décadas, y a raíz de los adelantos en el área de la $\mathrm{Re}$ producción Humana, le ha correspondido al campo de la esterilidad uno de los mayores progresos; es por ello que la Asociación Antioqueña de Obstetricia y Ginecología ha acogido con beneplácito la elección que se le ha hecho para la presentación de este relato.

Aunque la esterilidad no es un problema prioritario en Salud Pública, sí es una situación a la cual se deben dar soluciones, tanto en las universidades como en instituciones médicas, pues incide en la felicidad conyugal, en el comportamiento social y familiar de la pareja, y aun en el rendimiento laboral de cada uno de los miembros que la constituyen.

En nuestro país aún no se han hecho estudios prospectivos de los factores responsables en la esterilidad, por lo cual hemos tenido interés en presentar nuestra experiencia. 


\section{Material y métodos}

Se presentan los resultados obtenidos por el grupo de Ginecología Endocrina y Reproducción Humana, durante el período de 3 años comprendido en tre el $1^{\circ}$ de enero de 1976 y el 31 de diciembre de 1978, elaborados con la misma metodología y con carácter prospectivo.

\section{Pacientes}

Las pacientes estudiadas con el diagnóstico de esterilidad se clasificaron en tres grupos:

a. Esterilidad primaria: pacientes que luego de un año de exposición sin empleo de métodos anticonceptivos, no han quedado en embarazo.

b. Esterilidad secundaria: pacientes que habiendo tenido un embarazo (parto o aborto) no han quedado en embarazo luego de un año de exposición y sin empleo de métodos anticonceptivos.

c. Abortadora habitual: pacientes que consultan con historia de 3 abortos sucesivos o 4 alternos.

Para el estudio de las pacientes se utilizó una misma metodología con el fin de valorar todos los factores en el mínimo tiempo.

\section{Esquema de estudio}

\section{ESTUDIO DE LA PAREJA ESTERIL PROGRAMA DE INVESTIGACION}

\section{-Primera consulta}

Día 12 del ciclo

\section{- Segunda consulta}

Día 26 del ciclo

\section{-Tercera consulta}

Sí...

1. Prueba postcoito negativa moco de buena calidad

2. PPC negativa moco de mala calidad

3. Ciclo ovulatorio en fase y prueba postcoito (PPC) positiva

4. Biopsia fuera de fase

5. Ciclo anovulatorio

6. Atrofia endometrial
Historia clínica de la pareja

Prueba postcoito

Análisis de moco

Citología funcional

Biopsia de endometrio

Temperatura basal

Citología funcional

Análisis del moco

Se solicita:

Espermograma

Succinato de estriol u omifin

Laparoscopia e histerosalpingografía

Nueva biopsia

Prolactina - 17 cetosteroides

Inducción de la ovulación

Determinaciones hormonales

Tuberculina-histero-laparoscopia

$\mathrm{RX}$ de tórax-cultivo $\mathrm{BK}$ 
Historia y examen clínico de la pareja: interrogatorio y examen general a ambos esposos, instrucciones sobre el programa de estudio al cual van a ser sometidos. Se requiere que sea una pareja con unión estable.

Prueba postcoito: se practica entre 6 y 8 horas postcoito; se analizan cantidad y calidad del moco, filancia y grado de cristalización, viscosidad, número de leucocitos y eritrocitos, número y calida de espermatozoides. Se califica como positiva si hay más de 5 espermatozoides vivos con movilidad progresiva en un campo de $45 x$. Si el moco es de mala calidad se hace terapia para mejorar su calidad antes de valorar la prueba.

\section{Biopsia de endometrio}

Se toma el día 26 de un ciclo regular, el $2^{\circ}$ a $3^{\circ}$ premenstrual si tiene historia de oligomenorreas, o en cualquier momento si está en amenorrea, descar. tando previamente embarazo. Durante el ciclo de la biopsia no se proscriben las relaciones sexuales pues no interfieren con el desarrollo ovular y antes por el contrario, creemos que las maniobras cervicales desencadenan cambios hormonales que mejoran la implantación. Presentamos pacientes que consultan por esterilidad y han quedado en embarazo durante el ciclo de la biopsia, la cual se toma de la pared lateral del útero.

La fecha real de la biopsia (fecha correciida) resulta del recuento regresivo desde la víspera de la menstruación siguiente a la biopsia, que se considera como el día 28 , hasta el día del procedimiento.

La fecha del ciclo corregida se compara con la fecha del endometrio informada por Anatomía Patológica 1. Cuando las fechas son equivalentes o tienen una diferencia de un día se dice que está en fase, lo que descarta un problema endocrino. Si la diferencia es de 2 o más días se considera desfasada y por ende compatible con un cuerpo lúteo insuficiente, fenómeno que si se repite en 2 ciclos sucesivos hace el diag. nóstico. En el cuerpo lúteo insuficiente también es posible ver un endometrio en diferentes áreas de maduración o en parches, que hemos denominado endometrio en mosaico. La biopsia de endometrio bien leída es el mejor método diagnóstico para la función endocrina (Fig. 1)

Figura No. 1

Fechada de endometrio

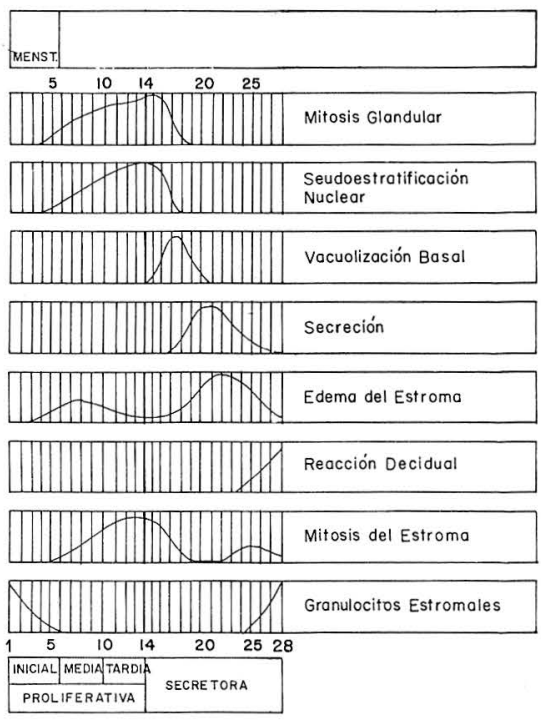

Noyes RW, Herting AT, Rock J.

Fertil.Steril 1:3,1950

En ocasiones se encuentran en la biopsia manifestaciones de infecciones crónicas o agudas por la presencia de plasmocitos, linfocitos, poliformos nucleares y aun lesiones caseosas con células de Langhans, que son indicios de un proceso infeccioso que ha pasado asintomático en las pacientes, pero que amerita un estudio cuidadoso para llegar a un diagnóstico etiológico. 
En la biopsia del día $26^{\circ}-27^{\circ}$ del ciclo se encuentran células que pueden ser confundidas con polimorfonucleares pero que recientemente se describen como granulocitos estromales. La atrofia endometrial es la manifestación de un hipoestrogenismo severo o el resultado de un proceso destructivo crónico tipo tuberculoso.

\section{Curva de temperatura basal}

Tomada por vía oral, todos los días. por dos meses sucesivos, luego de 6 a 8 horas de reposo en cama, se obtiene una gráfica que sufre modificaciones según la producción o no de progesterona, pues esta hormona o su metaboli. to, tienen propiedades termogénicas, actuando directamente sobre el centro térmico en el hipotálamo.

Hemos encontrado múltiples variaciones de la temperatura basal, principalmente en el cuerpo lúteo insuficiente (Fig. 2).

\section{Citología funcional}

Se toma los días $12^{\circ}$ y $26^{\circ}$ del ciclo, de las paredes laterales de la vagina; se aprecian variaciones de la citología de acuerdo con el influjo hormonal: elevada picnosis $(40$ a $50 \%)$ en la fase pre-ovulatoria, a un predominio de células intermedias (80 a 90\%) agrupa. das, adosadas, plegadas y con escasos leucocitos, en la fase progestacional.

Picnosis persistentes en los estados de anovulación; células intermedias, parabasales y basales en los diferentes grados de hipoestrogenismo o diferentes grados de androgenización. Para dar crédito a la citología funcional se debe descartar todo proceso infeccioso vaginal.

\section{Espermograma}

Se solicita cuando:

a. La prueba postcoito es negativa en 2 ocasiones sucesivas.
Figura No. 2

Cuerpo Lúteo Insuficiente, Temperatura Basal
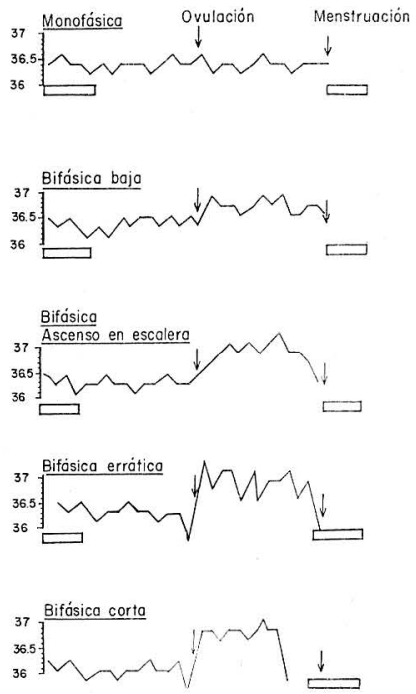

b. En pacientes con antecedentes o nico y patología genital al examen cli-

c. Ultimamente a todos los esposos al iniciarse el estudio de la pareja con el fin de motivarlos a colaborar.

El espermograma es tomado por masturbación o juego conyugal, en el hogar. luego de 4-6 días de abstinencia sexual, depositado en un recipiente apropiado y llevado al laboratorio en un tiempo menor de $1 / 2$ hora, evitando cambios bruscos de temperatura. Los espermogramas fueron realizados en 2 laboratorios donde tienen estandarizadas las técnicas y normalizadas las variables analizadas.

Se valoró la normalidad de un espermograma de acuerdo con los siguientes 
criterios obtenidos del análisis de 500 espermogramas realizados en Profamilia (Medellín 11).

1. Volumen de $2-5 \mathrm{cc}$

2. Concentración - más de 40 millones de espermatozoides/cc

3. Movilidad inicial: más del $60 \%$

4. Movilidad a las 2 horas: más del $40 \%$

5. Normalidad espermática: más del $80 \%$

Se califica un espermograma como anormal si presenta 2 o más variables alteradas, o una sola si está suficientemente alterada.

\section{Laparoscopia diagnóstica}

Se solicita laparoscopia diagnóstica:

1. Cuando todos los factores de esterilidad son normales.

2. Cuando luego de 3 ciclos ovulatorios inducidos, no ha quedado en embarazo.

3. Como examen inicial en el estudio de esterilidad en pacientes con antecedentes de:
a. Tuberculosis
b. Enfermedad inflamatoria pélvica
c. Dolor pélvico crónico
d. Patología pélvica al examen físico
e. Edad avanzada de la paciente
f. Varios años de estudio de esterili- dad previamente tratados
g. Cirugía abdominal previa.

Se practica el día $26^{\circ}$ del ciclo, con el fin de ver la presencia o no de cuerpo lúteo, núcleos de endometriosis, adherencias peritubáricas o periováricas, obstrucción tubárica por cromatoscopia y alteraciones morfológicas del útero y se complementa con biopsia de endometrio.
Histerosalpingografía: se solicita como complemento, si se encuentra alguna patología al estudio laparoscópico o si al tomar la biopsia de endometrio, se sospecha alguna patología intrauterina. Se toma en la fase folicular, con una buena tracción del cuello y sin espéculo, para evitar las posiciones anormales del útero; además, el medio de contraste debe ser en cantidad y a presión adecuada. Sólo son necesarias cuatro placas de buena calidad para el diagnóstico. En los casos de incompetencia istmico-cervical se toma el día 12 del ciclo, con un dedo del guante adherido distalmente a la cánula de Rubin y con tracción sostenida contra el cuello para luego medir el grado de dilatación cervical.

\section{Cirugía tubárica}

Se practicó incisión de Pfannenstiel en la mayoría de las pacientes intervenidas. El manejo de los tejidos y la hemostasia fueron muy cuidadosos, utilizando electrocauterio e instrumental de microcirugía para la liberación de adherencias, irrigación permanente del área quirúrgica con suero salino y aspiración continua; se prohibe la utilización de compresas y disección roma. Se peritonizaron todas las áreas cruentas y se hizo suspensión uterina a nivel de los ligamentos redondos, con material reabsorvible, en aquellos casos en que no fue posible peritonizar todas las áreas cruentas localizadas principalmente en el douglas. Se practicó cromatoscopia intra-operatoria e hidrotubación por 5 a 7 días en el postoperatorio. con $30 \mathrm{cc}$ de suero salino y $50 \mathrm{mg}$ de hidrocortisona.

Se aplicaron antibióticos postoperatorios por vía oral.

\section{Determinaciones, hormonales}

Son de gran utilidad práctica para llegar a un diagnóstico etiológico y complementan los resultados obtenidos en el estudio de los efectores (Figs. 3, 4 y 5). 
Figura No. 3

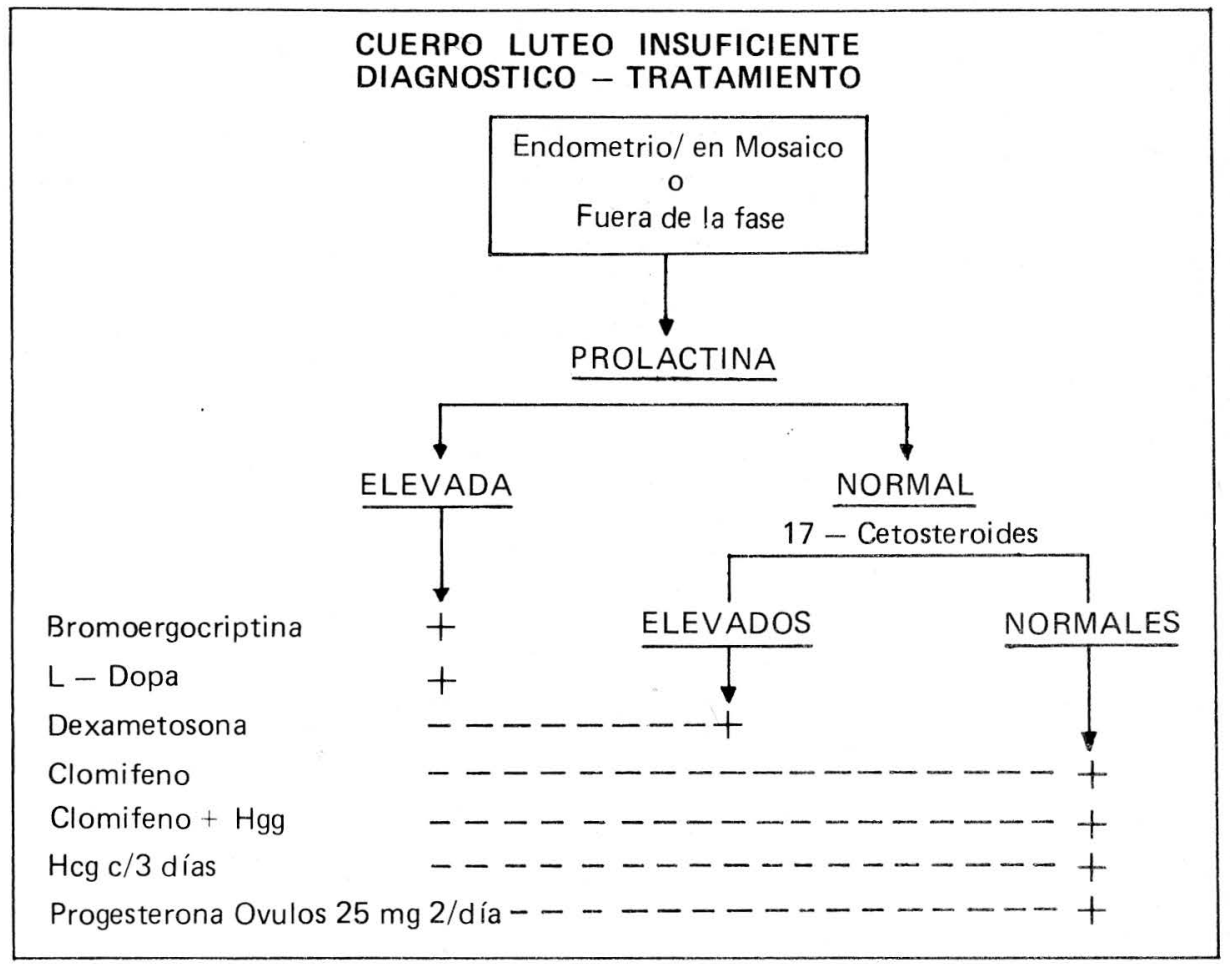

La FSH-LH y Prolactina determinadas entre el $6^{\circ}$ al $10^{\circ}$ día del ciclo, son de gran utilidad en los trastornos endocrinos tales como cuerpo lúteo insuficiente, anovulación, y principalmente, en el hipoestrogenismo para determinar la glándula alterada y la severidad del daño.

Sin embargo, en los casos de anovulación la biopsia de endometrio es suficiente para el diagnóstico, correlacionada con el resto de factores, pues las determinaciones de gonadotropinas se encuentran siempre alteradas: disminución de FSH y LH normal o elevada, por lo cual hemos disminuido la solicitud de estos exámenes. No se practicaron pruebas de reserva hipofisiaria.
Por la imposibilidad actual de determinar en nuestro medio esteroides por RIA y principalmente 17 hidroxiprogesterona, androstenodiona y sulfato de Dehidroepiandrosterona, aún utilizamos la determinación de 17 Cetosteroides en orina de 24 horas, siendo $15 \mathrm{mg}$ en 24 horas el límite superior normal, por encima del cual se hacen pruebas de supresión durante un mes con dosis ascendentes de dexametasona, a las $11 \mathrm{p}$. $\mathrm{m}$. hasta lograr valores por debajo de $10 \mathrm{mg}$ en 24 horas.

\section{Resultados}

Se informan los resultados del estudio de 229 parejas; $27(11.8 \%)$ son cla- 
Figura No. 4

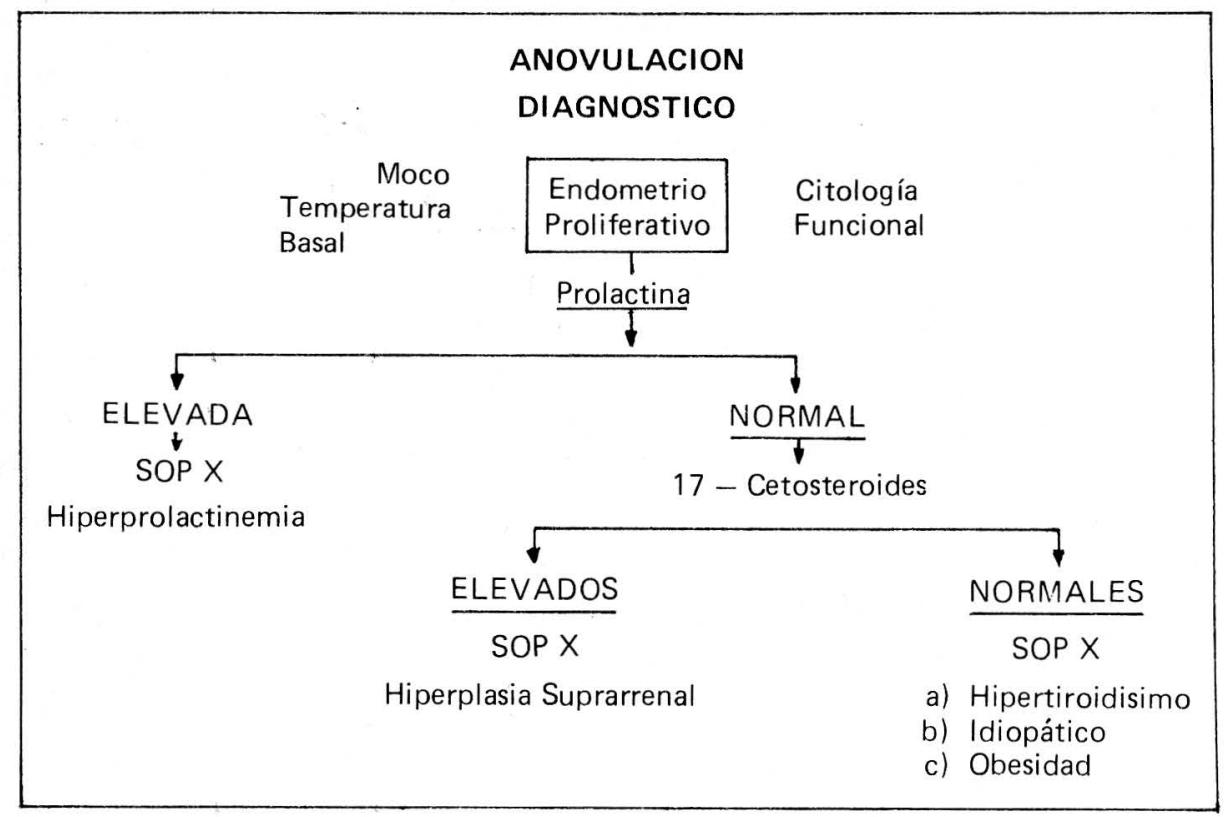

sificadas como abortadoras habituales y el resto, 202, son estérilès, $134(66 \%)$ primarias y $68(34 \%)$ secundarias (Cuadro 1).

\section{CUADRO N: 1}

Distribución de 229 pacientes con trastornos de la fertilidad, según edad y tipo

\begin{tabular}{crrrr}
\hline Edad & $\begin{array}{c}\text { Esterilidad } \\
\text { primaria }\end{array}$ & $\begin{array}{c}\text { Esterilidad } \\
\text { secundaria }\end{array}$ & $\begin{array}{c}\text { Abortadora } \\
\text { habitual }\end{array}$ & Total \\
\hline-20 & $14(10.4)$ & $4(5.9)$ & $1(3.7)$ & $19(8.3)$ \\
$21-25$ & $41(30.6)$ & $19(30.0)$ & $10(37.0)$ & $70(30.6)$ \\
$26-30$ & $47(35.0)$ & $22(32.3)$ & $5(18.5)$ & $74(32.3)$ \\
$31-35$ & $27(20.0)$ & $20(29.4)$ & $10(37.0)$ & $57(24.9)$ \\
+36 & $5(3.8)$ & $3(4.4)$ & $1(3.7)$ & $9(4.0)$ \\
\hline Total & $134(100)$ & $68(100)$ & $27(100)$ & $229(100)$ \\
\hline
\end{tabular}


Figura No. 5

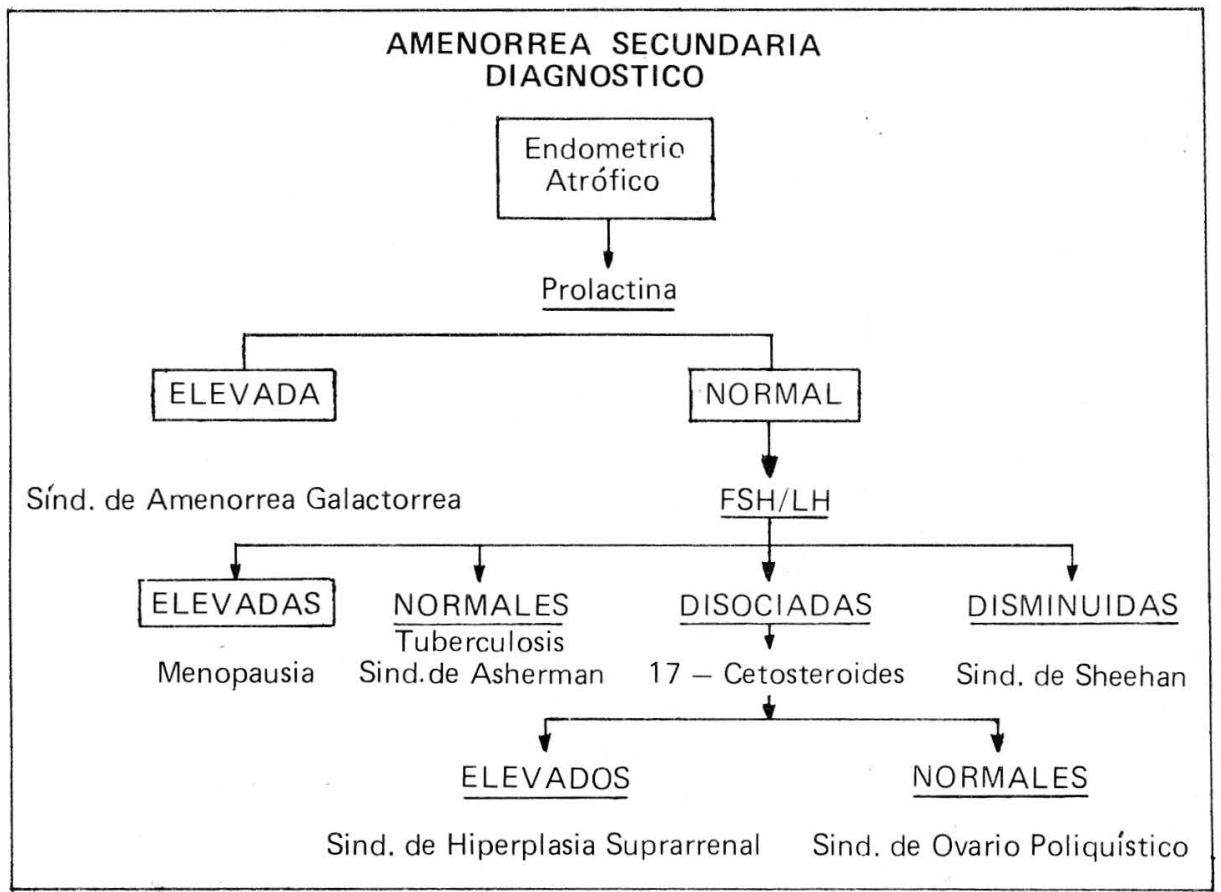

Edad: el mayor grupo correspondió a pacientes entre 26-30 años, 47 pacientes $(35 \%)$ en la esterilidad primaria y 22 $(32.3 \%)$ en la secundaria; en el grupo de abortadoras habituales hubo 2 grupos mayoritarios, entre 21-25 y 31-35 años con 1.0 pacientes cada uno $(37 \%)$.

Si se analizan los años de esterilidad en las 202 parejas estudiadas, se puede apreciar cómo nuestras pacientes consultan tardíamente, cuando tienen 40 más años de esterilidad, el 56 a $58 \%$ por esterilidad primaria y secundaria respectivamente, siendo el porcentaje mayor para el grupo de más de 6 años 32 y $38.2 \%$ hecho que coincide con la avanzada edad de las pacientes en el momento de la consulta (Cuadro 2).

Estado civil: 206 parejas $(89.9 \%$ son casadas y sólo $23(10.1 \%)$ están clasi- ficadas como unión libre estable; esto es el resultado del requisito que se exige a las parejas para ser estudiadas (Cuadro 3).

\section{Factores etiológicos y su frecuencia en la esterilidad}

Todas las parejas estudiadas fueron clasificadas de acuerdo con el factor de esterilidad con el fin de planear un tratamiento etiológico.

Al factor endocrino le correspondió ser la primera causa de esterilidad, 122 pacientes $(60.4 \%)$, de las 202 pacientes estudiadas, siendo uno de los porcentajes más altos informados en la literatura, como consecuencia posiblemente del mayor diagnóstico de cuerpo lúteo insuficiente e hiperplasia suprarenal. 
CUADRO № 2

Distribución de 202 pacientes, según los años de esterilidad

\begin{tabular}{rrrr}
\hline Años & $\begin{array}{c}\text { Esterilidad } \\
\text { primaria }\end{array}$ & $\begin{array}{c}\text { Esterilidad } \\
\text { secundaria }\end{array}$ & Total \\
\hline 1 & $17(12.8)$ & $8(11.7)$ & $25(12.3)$ \\
$2-3$ & $41(30.6)$ & $20(29.0)$ & $61(30.2)$ \\
$4-5$ & $33(24.6)$ & $14(20.6)$ & $47(23.3)$ \\
+6 & $43(32.0)$ & $26(38.2)$ & $69(34.2)$ \\
\hline Total & $134(100.0)$ & $68(100.0)$ & $202(100.0)$ \\
\hline
\end{tabular}

CUADRO N: 3

Distribución de 229 pacientes estériles, según el estado civil

\begin{tabular}{|c|c|c|c|c|}
\hline & $\begin{array}{l}\text { Esterilidad } \\
\text { primaria }\end{array}$ & $\begin{array}{l}\text { Esterilidad } \\
\text { secundaria }\end{array}$ & $\begin{array}{l}\text { Abortadora } \\
\text { hahitual }\end{array}$ & Total \\
\hline $\begin{array}{l}\text { Casadas } \\
\text { Unión libre estable }\end{array}$ & $\begin{array}{r}127 \\
7\end{array}$ & $\begin{array}{l}55 \\
13\end{array}$ & $\begin{array}{r}24 \\
3\end{array}$ & $\begin{array}{r}206(89.9) \\
23(10.1)\end{array}$ \\
\hline Total & 134 & 68 & 27 & $229(100.0)$ \\
\hline
\end{tabular}

Las entidades endocrinas más frecuentes son en su orden, el síndrome de ovario androgénico idiopático con un $53.3 \%$, el cuerpo lúteo insuficiente $26.2 \%$, la hiperplasia suprarrenal congénita $10.6 \%$ y los estados de hiperprolactinemia $8 \%$, predominando el factor endocrino en las pacientes con esterilidad primaria (Cuadro 4).

El síndrome de anovulación crónica, síndrome de ovario poliquístico o síndrome de ovario androgénico (el cual creemos sea el más descriptivo), se clasificó de acuerdo con la causa etiológica en: a) Idiopático. b) Hiperplasia suprarrenal. c) Obesidad. d) Hiperprolactinemia y e) Hipertiroidismo, este último grupo de pacientes consultan más al internista que al ginecólogo. El trata- miento es diferente de acuerdo con la causa etiológica; esto hace que sea tan importante un estudio exhaustivo con el fin de obtener mejores resultados.

Se trataron 37 pacientes $(56.9 \%)$ de las 65 estudiadas con el diagnóstico de síndrome de ovario poliquístico, 20 pacientes $(54 \%)$ quedaron en embarazo, 11 a término, 1 aborto, 1 embarazo ectópico, 2 en consulta prenatal y 5 sin datos del embarazo, luego de 4 meses de control prenatal (Cuadro 5).

En 35 pacientes se utilizó el citrato de clomifeno a dosis ascendentes por ciclo, si no se obtenía la ovulación; en 33 pacientes $(94 \%)$ se detectó ovulación y $18(51.4 \%)$ se embarazaron. Una paciente quedó en embarazo a los $2 \mathrm{me}$ - 
CUADRO Nㄴ 4

Frecuencia y distribución en 202 parejas estériles por causa endocrina

\begin{tabular}{lrrr}
\hline & $\begin{array}{c}\text { Esterilidad Esterilidad } \\
\text { primaria }\end{array}$ \\
& & & \\
& & & \\
& & & \\
& & & \\
Síndrome ovario poliquístico & 40 & 25 & $53.3)$ \\
Hiperplasia suprarrenal & 10 & 3 & 13 \\
SOP e hiperprolactinemia & 5 & 0 & 5 \\
Amenorrea e hiperprolacíinemia & 5 & 0 & 5 \\
Cuerpo lúteo insuficiente & 21 & 11 & $32(26.2)$ \\
Menopausia precoz & 1 & - & $i$ \\
Disgenesla gonadal en mosaico & 1 & - & 1 \\
\hline Total & 83 & 39 & $122(100 \%)(60.4)$ \\
\hline
\end{tabular}

CUADRO № 5

Sindrome de ovario poliquístico - Resultados citrato de clomifeno

\begin{tabular}{|c|c|c|c|c|c|c|c|c|c|}
\hline Esterilidad & $\begin{array}{l}\mathrm{N} \text { : total de } \\
\text { pacientes }\end{array}$ & $\begin{array}{c}\mathrm{N}: \text { de } \\
\text { pacientes }\end{array}$ & Ovulación & Embarazos & $\begin{array}{c}\mathrm{A} \\
\text { término }\end{array}$ & Abortos & Ectópicos & Prenatc & $\begin{array}{c}\text { Sin } \\
\text { I datos }\end{array}$ \\
\hline Primaria & 40 & 22 & 19 & 13 & 6 & 1 & 1 & 2 & 3 \\
\hline Secundaria & 25 & 13 & 13 & 5 & 3 & 0 & 0 & - & 2 \\
\hline & $\overline{65}$ & $\overline{35}$ & $\overline{32}(91.4 \%)$ & $\overline{18}$ & $\overline{9}$ & - & - & - & $\overline{5}$ \\
\hline Pos omifin & b5 & 1 & - & 1 & 1 & 0 & 0 & - & 0 \\
\hline de peso & - & 1 & - & 1 & 1 & 0 & 0 & - & 0 \\
\hline Total & 65 & 37 & 32 & $20(54 \%)$ & $11(55 \%$ & о) 1 & 1 & 2 & 5 \\
\hline
\end{tabular}

ses de suspender el clomifeno y 1 al disminuir 30 kilos de peso (Cuadro 6)

Fueron tratadas 25 pacientes de las 32 estudiadas con el diagnóstico de cuerpo lúteo insuficiente obteniéndose 18 embarazos, 13 a término, 3 abortos, 1 embarazo ectópico y 1 paciente en control prenatal. Diez de los embarazos ocurrieron luego de procedimientos diagnósticos, 6 de los cuales postbiopsia de endometrio, 7 con tratamiento médico: citrato de clomifeno, pues aún no utilizábamos los óvulos de progesterona y una paciente durante el período de estudio (Cuadro 7).
En tercer lugar la hiperplasia suprarenal; 12 pacientes fueron tratadas con dexametasona a dosis supresivas, hubo 5 embarazos de los cuales 4 fueron a término.

En los estados de hiperprolactinemia con síndrome de ovario poliquístico, de 5 pacientes tratadas hubo sólo 2 embarazos con bromoergocriptina; los otros 3 , tratados con L-Dopa mostraron resultados negativos; en el síndrome de amenorrea galactorrea sólo hubo un embarazo con L-Dopa.

Al factor tuboperitoneal le correspondió el segundo lugar en frecuencia, 88 


\section{CUADRO N: 6}

\section{Esquema utilizado de citrato de clomifeno y respuesta ovulatoria en 35 pacientes con síndrome de ovario poliquístico}

\begin{tabular}{ccccc}
\hline Esquema & $\begin{array}{c}\text { No total de } \\
\text { pacientes }\end{array}$ & $\begin{array}{c}\text { No pacientes } \\
\text { ovularon }\end{array}$ & Porcentaje & Embarazos \\
\hline $50 \times 5$ & 35 & 20 & 57 & $18(51.4)$ \\
$100 \times 5$ & 15 & 7 & 20 & \\
$50 \times 10$ & 8 & 4 & 11.5 & \\
$50 \times 10$ HCG (5.000 UI) & 2 & 2 & 5.75 & \\
No ovularon & 2 & 2 & 5.75 & \\
\hline
\end{tabular}

Número total de pacientes con cuerpo lúteo insuficiente y resultados obtenidos

\begin{tabular}{|c|c|c|c|c|c|c|c|}
\hline & $\begin{array}{l}\mathrm{N} \text { total de } \\
\text { pacientes }\end{array}$ & $\begin{array}{l}\mathrm{N} \text { de pacien- } \\
\text { tes tratados }\end{array}$ & $\stackrel{N}{\text { embarazos }}$ & $\stackrel{A}{\text { término }}$ & Abnrtos & Ectópico & Prenatal \\
\hline \multicolumn{8}{|l|}{ Esterilidad } \\
\hline Primaria & 21 & 16 & & & & & \\
\hline \multirow{2}{*}{\multicolumn{5}{|c|}{ Tratamiento }} & & & \\
\hline & & & & 2 & 1 & - & - \\
\hline Dex omitin & & & 3 & 3 & 一 & - & - \\
\hline \multicolumn{8}{|l|}{ Pos biopsia $1^{\circ}$} \\
\hline $2^{\circ}$ & & & 2 & - & 1 & & 1 \\
\hline Pos HSG & & & 1 & 1 & & & \\
\hline \multicolumn{8}{|l|}{ Pos laparoscopia } \\
\hline $1 \stackrel{0}{\circ}$ & & & 2 & 1 & & & \\
\hline $2^{\circ}$ & & & 1 & 1 & 1 & & \\
\hline En el estudio & & & 1 & 1 & & & \\
\hline Total & 32 & $25(78)$ & $18(72)$ & 13 & 3 & 1 & 1 \\
\hline
\end{tabular}

pacientes $(43.6 \%)$ de las 202 estudiadas (Cuadro 8).

Las causas etiológicas más frecuentes en el factor tuboperitoneal, son las secuelas de procesos sépticos: 60 pacientes $(60.2 \%), 22$ por tuberculosis genital $(10.8 \%)$ de las 202 pacientes y el resto, 38 pacientes $(18.8 \%)$ se clasificaron como secuelas de procesos sépticos inespecíficos. (Cuadro No. 9).
En el segundo lugar las secuelas de cirugías abdominales previas, principalmente en la pelvis, 18 pacientes $(9 \%)$; por resección cuneiforme de ovario en 7 pacientes $(7.8 \%)$ de las 88 pacientes con factor tuboperitoneal, $0 \quad 38.8 \%$ de las cirugías pélvicas.

En último lugar la endometriosis, 10 pacientes $(5 \%)$ del total de pacientes estériles o $14.4 \%$ del factor tuboperito- 
CUADRO N $N 8$

FACTOR TUBOPERITONEAL

Frecuencia y procedimientos quirúrgicos en 202 pacientes estériles

\begin{tabular}{lrl}
\hline & No pacientes & Procedimientos quirúrgicos \\
\hline Pacientes estudiadas & $202(100 \%)$ & \\
Factor tuboperitoneal & $88(43.6 \%)$ & Terapéuticos
\end{tabular}

CUADRO N: 9

FACTOR TUBOPERITONEAL

Distribución por causas etiológicas en 88 pacientes con factor tuboperitoneal Estudio de 202 pacientes estériles

\begin{tabular}{lll}
\hline & $\mathrm{N}^{\circ}$ pacientes & $\%$ \\
\hline Inespecífico & 38 & 43.1 \\
Tuberculosis & $22(10.8 \%)$ & 25 \\
Postquirúrgico & 18 & 20.4 \\
Endometriosis & 10 & 11.36 \\
\hline Total & $88(43.5 \%)$ & 100 \\
\hline
\end{tabular}

neal, el cual se hizo de acuerdo con la clasificación de Acosta modificada por Kistner 12 (Cuadro 10).

Las pacientes con el diagnóstico de TBC (22 pacientes) se trataron sólo médicamente si tenían el proceso agudo, a ninguna se le hizo tratamiento quirúrgico. Luego del total de 88 pacientes con factor tuboperitoneal se intervinieron 44 pacientes, 5 con fines paliativos para tratar el dolor pélvico; cirugía reparadora o terapéutica a 39 pacientes; el resto de pacientes no se intervinieron por ser de mal pronóstico o por problemas personales o familiares.

Los procedimientos realizados fueron:
Salpingolisis en 8 pacientes, salpingoplastia sola o con salpingolisis en 21 y resección y electrofulguración de endometriosis en 9 (Cuadro 11).

El seguimiento de las 39 pacientes intervenidas fue de 1 a 3 años; hubo 12 embarazos $(30.7 \%)$, de los cuales 5 llegaron a término, 6 embarazos ectópicos y un aborto (Cuadro 12).

Correspondiendo al grupo de salpingolisis $\mathrm{y} / \mathrm{o}$ electrofulguración de endometriosis el $29.4 \%$ y, el $33 \%$ al grupo de salpingoplastia, teniendo la mayor frecuencia de embarazos ectópicos.

EI factor masculino se evaluó analizando los resultados de la prueba post- 


\section{CUADRO N 10}

FACTOR TUBOPERITONEAL

Antecedentes quirúrgicos en 88 pacientes con factor tuboperitoneal

\begin{tabular}{lcr}
\hline \multicolumn{1}{c}{ Procedimiento } & N pacientes & $\%$ \\
\hline Salpingectomía por E. ectópico & 10 & 11.3 \\
Resección en cuña de ovaric & 7 & 7.9 \\
Anexectomía & -3 & 3.4 \\
Apendicectomía & 3 & 3.4 \\
Cesárea & 1 & 1.1 \\
Miomectomía & 1 & 71.5 \\
Ninguno & 63 & 100 \\
\hline Total & 88 & \\
\hline
\end{tabular}

CUADRO N: 11

FACTOR TUBOPERITONEAL.

Distribución de procedimientos quirúrgicos y embarazos en 39 pacientes estériles

\begin{tabular}{lcc}
\hline \multicolumn{1}{c}{ Procedimiento } & № pacientes & № embarazos \\
\hline Salpingolisis & $8(20.5 \%)$ & $3(37.5 \%)$ \\
Salpingolisis y/o & $21(53.8 \%)$ & $7(33.3 \%)$ \\
Salpingoplastia & $9(23 \%)$ & $2(22.2 \%)$ \\
$\begin{array}{l}\text { Resección y/o electrofulguración } \\
\text { de endometriosis }\end{array}$ & $1(2.5 \%)$ & \\
Neoimplantación & $39(100 \%)$ & $12(30.7 \%)$ \\
\hline Total & & \\
\hline
\end{tabular}

CUADRO N: 12

\section{FACTOR TUBOPERITONEAL}

Evolución de los embarazos, según procedimientos quirúrgicos en 39 pacientes

\begin{tabular}{lccccc}
\hline & $\begin{array}{c}\mathrm{N}^{\circ} \\
\text { bacientes }\end{array}$ & $\begin{array}{c}\mathrm{N} \text { e } \\
\text { embarazos }\end{array}$ & $\begin{array}{c}\mathrm{N}^{\circ} \\
\text { abortos }\end{array}$ & $\begin{array}{c}\mathrm{N}: \\
\text { ectópicos }\end{array}$ & $\begin{array}{c}\mathrm{N} \text { : } \\
\text { embarazos a término }\end{array}$ \\
\hline $\begin{array}{l}\text { Salpingolisis y/o } \\
\text { electrofulguración } \\
\text { endometriosis }\end{array}$ & 17 & $5(29.4 \%)$ & 0 & 1 & $4(23.5 \%)$ \\
$\begin{array}{l}\text { Salpingolisis y/o } \\
\text { salpingoplastia }\end{array}$ & 21 & $7(33.3 \%)$ & 1 & 5 & $1(4.7 \%)$ \\
$\begin{array}{l}\text { Neoimplantación } \\
\text { Total }\end{array}$ & 1 & 0 & 0 & 0 & 0 \\
\hline
\end{tabular}


coito y del espermograma. Se estudiaron 107 hombres $(53 \%$ del total de 202 parejas), la mayoría de ellos con pruebas postcoito negativas.

La edad de los pacientes osciló entre 22 y 60 años y con un promedio de 4 a 5 años de vida sexual activa (VSA); sólo 14 pacientes $(13.1 \%)$ pertenecian a la esterilidad secundaria (Cuadro 13).

De los 107 espermogramas estudiados, $53(49.5 \%)$ tenian un espermograma alterado, lo que representa el $26.2 \%$ del total (Cuadro 14).
En 28 parejas $(13.9 \%)$ se encontró un doble factor de esterilidad o factor mixto; luego, el factor masculino como causa única es de sólo $12.3 \%$.

En relación con la alteración en los índices del espermograma: 31 pacientes $(30.4 \%)$ tuvieron trastornos en el volumen, 40 pacientes $(39.6 \%)$ tuvieron alteración en la concentración espermática. Es de resaltar que 12 pacientes (6\% de las 202 parejas y $11.9 \%$ de 107 pacientes estudiados) eran azoospérmicos (Cuadro 15).

\section{CUADRO N: 13}

\section{Distribución de la esterilidad masculina en 107 pacientes}

\begin{tabular}{ccc}
\hline Factor & N: pacientes & $\%$ \\
\hline Primaria & 65 & 60.7 \\
Secundaria & 14 & 13.1 \\
Mixto & 28 & 26.2 \\
\hline
\end{tabular}

\section{CUADRO $\mathrm{N}: 14$}

Estudio de la pareja estéril - Factor masculino

\begin{tabular}{lcc}
\hline Espermograma & N: pacientes & $\% 202$ pacientes \\
\hline Normales & 50 & 24.8 \\
Anormales & 53 & $\frac{26.2}{2.0}$ \\
Sin datos & 4 & 2.0 \\
\hline Total & 107 & 53.0 \\
\hline
\end{tabular}

\section{CUADRO № 15}

Concentración de espermatozoides por cc en 101 pacientes

\begin{tabular}{rccc}
\hline Millones cc & No pacientes & $\%$ & $\begin{array}{c}\text { Promedio } \\
\text { millones cc }\end{array}$ \\
\hline 0 & 12 & 11.9 & 0 \\
$1-39$ & 28 & 27.7 & 16.8 \\
$>40$ & 61 & 60.4 & 88.9 \\
\hline Total & 101 & 100.0 & $66.2^{*}$ \\
\hline
\end{tabular}

* Sin incluir azoospérmicos. 
Se puede apreciar en los cuadros 16 , 17 y 18 la normalidad y los grados de movilidad inicial y a las 2 horas, en los cuales el espermograma está alterado en un $30.9,29.2$ y $32.9 \%$ respectivamente.

CUADRO N: 16

Normalidad espermákica en 84 pacientes

\begin{tabular}{lccc}
\hline$\%$ promedio & No pacientes & $\%$ & $\begin{array}{c}\text { Normales } \\
\text { promedio } \%\end{array}$ \\
\hline 80 y más & 58 & 69.1 & 88.5 \\
Menos de 80 & 26 & 30.9 & 59.7 \\
\hline Total & 84 & 100.0 & 79.6 \\
\hline
\end{tabular}

CUADRO № 17

Movilidad espermática inicial en 89 pacientes

\begin{tabular}{lccc}
\hline$\%$ movilidad & No pacientes & $\%$ & $\begin{array}{c}\text { Movilidad } \\
\text { promedio } \%\end{array}$ \\
\hline 60 y más & 63 & 70.8 & 76.3 \\
Menos de 60 & 26 & 29.2 & 33.7 \\
\hline Total & 89 & 100.0 & 63.9 \\
\hline
\end{tabular}

CUADRO № 18

Movilidad espermática a las 2 horas en 82 pacientes

\begin{tabular}{lccc}
\hline$\%$ movilidad & No pacientes & $\%$ & $\begin{array}{c}\text { Movilidad } \\
\text { promedio } \%\end{array}$ \\
\hline 40 y más & 55 & 67.7 & 53.4 \\
Menos de 40 & 27 & 32.9 & 16.9 \\
\hline Total & 82 & 100.0 & 41.4 \\
\hline
\end{tabular}

\section{Factor cervical}

Es con el factor útero vaginal, uno de los factores menos frecuentes; su valoración se hizo indirectamente por medio de la prueba postcoito, pues no contamos con métodos adecuados para el estudio de procesos infecciosos específi- cos como el T Micoplasma o la Clamidia Trascomatis o estudios inmunológicos. De 11 pacientes con prueba postcoito negativa y espermograma normal, en $5(7.8 \%)$ había una causa endocrina que posiblemente alteraba lia calidad del moco y en $6(9.4 \%)$ era de causa desconocida, posiblemente el factor cervical puro (Cuadro 19). 
Cuadro No. 19

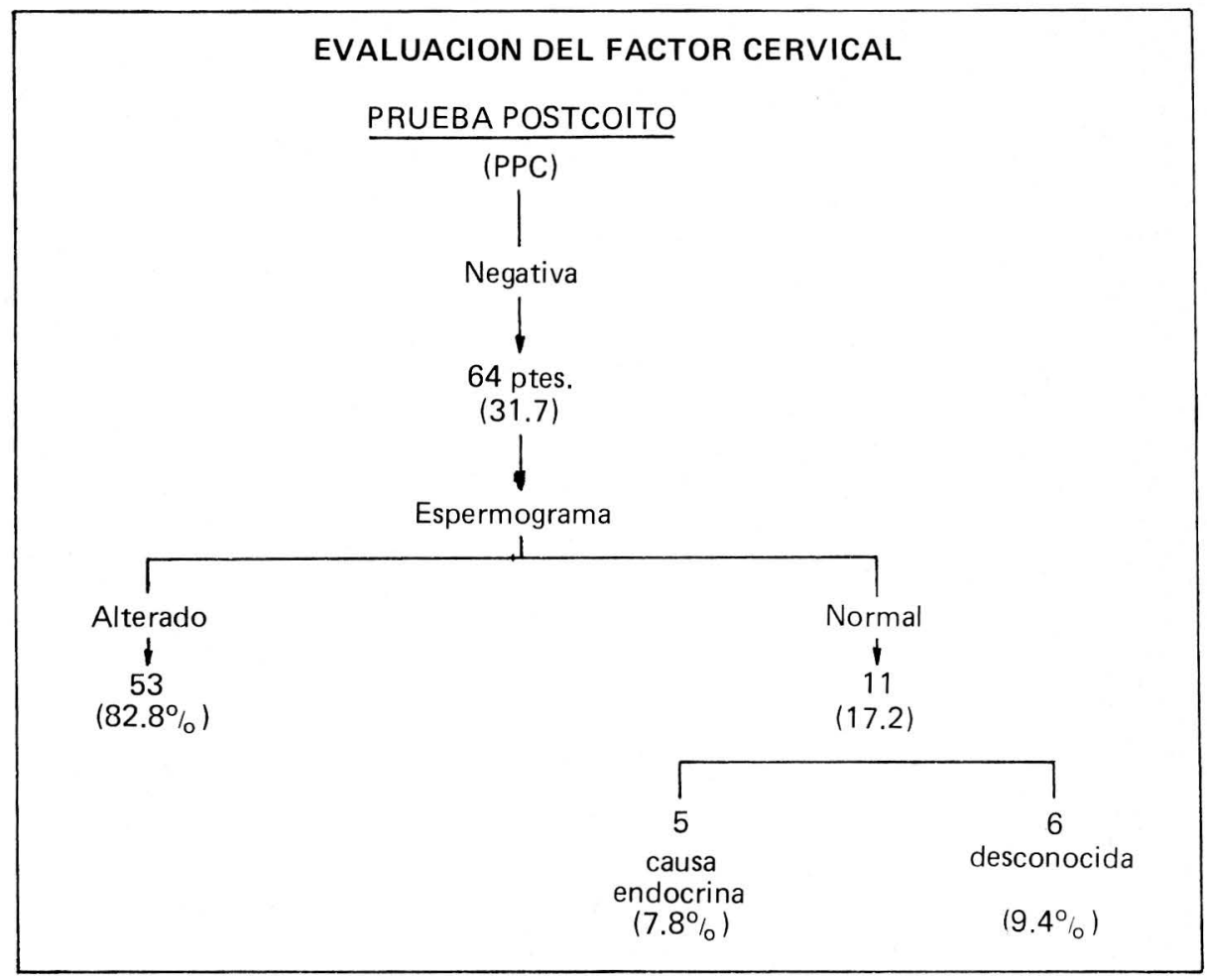

El factor uterino y el coito-vaginal tuvieron la menor frecuencia como causa primaria de esterilidad. Se excluyeron del factor uterino los pacientes con secuelas de tuberculosis genital, los cuales se incluyeron al grupo de alteración del factor tuboperitoneal, por tanto sólo se encontraron 3 pacientes: un síndrome de Rockitansky cuyo motivo de consulta fue más por la esterilidad que por su amenorrea primaria; un síndrome de Asherman y una paciente con doble útero, la cual quedó en embarazo luego del tratamiento quirúrgico. Una paciente se clasificó en el grupo coito-vaginal, la cual quedó en embarazo al resecár. sele el septum vaginal transverso, el cual tenía un pequeño orificio por donde menstruaba luego de una persistente dismenorrea.

\section{Esterilidad de causa desconocida}

Comprende un total de 11 parejas, una de ellas clasificada en el grupo de causa idiopática, a la cual no se le encontró ninguna causa de su esterilidad; 10 parejas ( $5 \%$ del total) clasificadas como normales quedaron en embarazo al ingreso o durante el período de estudio de su esterilidad, sin lograrse detectar ninguna causa etiológica. 
Con los adelantos en los métodos diagnósticos, la frecuencia de esterilidad de causa idiopática es cada vez menor, es así como en nuestra investigación casi siempre se llegó al diagnóstico de la causa etiológica de la esterilidad.

\section{Abortadora habitual}

Se da un corto informe de los resultados obtenidos, pues será tema de otra publicación.

De las 27 parejas estudiadas, 11 $(40.7 \%)$ tenían cuerpo lúteo insuficiente, $6(22.2 \%)$ incompetencia ístmico cervical, y 10 sin diagnóstico definido, 7 de las cuales Ilegaron en embarazo o lo hicieron durante el estudio y a sólo
3 pacientes no se les encontró una causa evidente de su esterilidad (Cuadro 20).

Del total de pacientes estudiadas 22 $(81.5 \%)$ quedaron en embarazo, 16 a término $(72.7 \%), 4$ abortos y 2 en consulta perinatal.

Es posible que en varias pacientes que se embarazaron al ingreso o luego de procedimientos quirúrgicos, se trate de cuerpos lúteos insuficientes como causa de aborto.

Se anexa el cuadro comparativo de los trabajos publicados sobre el estudio de la pareja estéril, en relación con nuestros resultados " 2 a 10". El de la doctora Jones Ges, es una revisión únicamente sobre parejas con esterilidad primaria (Cuadro 21).

CUADRO N:20

Análisis de 27 pacientes con diagnóstico de aborto habitual

\begin{tabular}{|c|c|c|c|c|c|}
\hline & $\begin{array}{c}\mathrm{N}^{\circ} \\
\text { pacientes }\end{array}$ & $\begin{array}{c}\mathrm{N}: \\
\text { embarazos }\end{array}$ & $\begin{array}{c}\mathrm{N}^{\circ} \\
\text { abortos }\end{array}$ & $\begin{array}{c}\text { En } \\
\text { prenatal }\end{array}$ & $\begin{array}{l}\text { Embarazos } \\
\text { a término }\end{array}$ \\
\hline \multicolumn{6}{|l|}{ Embarazos al } \\
\hline ingreso (sin datos) & 4 & 4 & 一 & - & 4 \\
\hline Embarazos en el & & & & & \\
\hline estudio (sin datos) & 2 & 2 & - & 1 & 1 \\
\hline $\begin{array}{l}\text { Embarazos post. } \\
\text { biopsia (C.L.I.) }\end{array}$ & 7 & 7 & 2 & - & 5 \\
\hline Cuerpo lúteo & & & & & \\
\hline $\begin{array}{l}\text { insuficiente } \\
\text { Incompetencia }\end{array}$ & 4 & 4 & $1^{*}$ & - & 3 \\
\hline ístmico cervical & $6^{*}$ & 4 & 1 & - & 3 \\
\hline $\begin{array}{l}\text { Embarazo post-histero } \\
\text { salpingografía }\end{array}$ & 1 & 1 & - & 1 & 0 \\
\hline Sin clasificar & 3 & - & - & - & - \\
\hline Total & 27 & 22 & 4 & 2 & 16 \\
\hline
\end{tabular}

* Utero bicorne 


\section{CUADRO № 21}

\section{Estudio de la pareja estéril - Resultados}

\begin{tabular}{lrrrrr}
\hline \multicolumn{1}{c}{ Autor } & $\begin{array}{c}\text { Parejas } \\
\text { estudiadas }\end{array}$ & $\begin{array}{c}\text { Factor } \\
\text { endocrino }\end{array}$ & $\begin{array}{c}\text { Factor } \\
\text { tubárico }\end{array}$ & $\begin{array}{c}\text { Factor } \\
\text { masculino }\end{array}$ & $\begin{array}{r}\text { Factor } \\
\text { cervical }\end{array}$ \\
\hline Jones GES. y col. & 555 & $121(21.8)$ & $131(23.6)$ & $81(14.6)$ & $20(3.6)$ \\
Dor J. y cols. & 665 & $133(20.0)$ & $68(10.2)$ & $120(18.0)$ & $6(0.9)$ \\
Delgado-UJ. y cols. & 500 & $77(15.0)$ & $211(42.2) *$ & $31(6.2)$ & $11(2.2)$ \\
Garcia A. y cols. & 479 & $160(32.4)$ & $164(34.2)$ & $85(17.7)$ & $\underline{143(29.8)}$ \\
Diaz-Intante y cols. & 204 & $69(33.8)$ & $30(14.7)$ & $11(5.3)$ & $29(14.2)$ \\
Peralta MT. y cols. & 232 & $31(13.5)$ & $41(17.7)$ & $60(25.9)$ & $56(24.1)$ \\
Sánchez F. y cols. & 202 & $\underline{122(60.4)}$ & $\underline{88(43.6)} *$ & $\underline{53(26.2)}$ & $6(2.9)$ \\
\hline
\end{tabular}

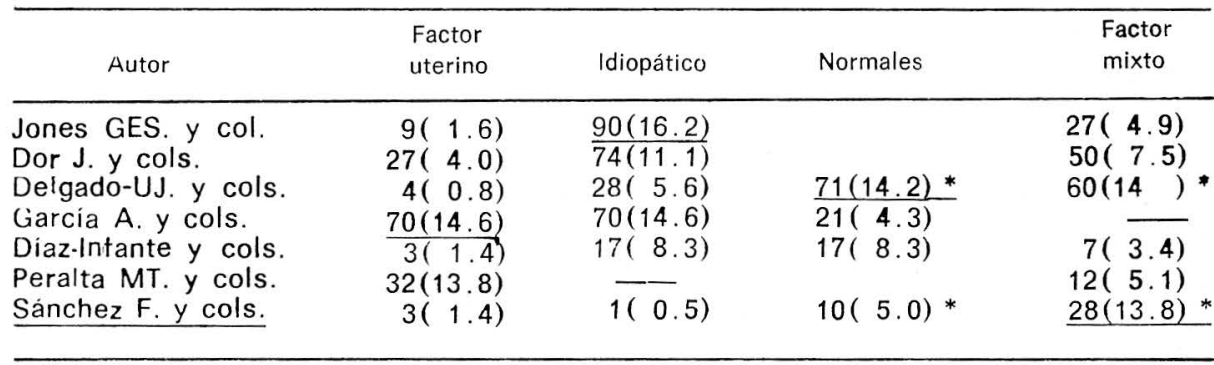

* Embarazadas en el estudio - prospectivo.

\section{DISCUSION}

La consulta de esterilidad es cada vez más importante, debido a los avances con la especialidad y al interés de las parejas en solucionar su problema.

Se calcula que en Medellín, con una población de 1.484 .000 habitantes, hay 38.600 personas estériles y 675.000 en el resto del país de una población aproximada de 26 millones de habitantes.

Se debe utilizar una metodología racional para el estudio de la paciente, que garantice un estudio rápido de la pareja, con el fin de programarle un plan terapéutico y de sequimiento adecuado, además de utilidad práctica para la enseñanza.
Siguiendo el mismo esquema de estudio para todas las pacientes se puede apreciar que el factor endocrino ocupó el primer lugar en frecuencia, siendo importante resaltar el mayor número de pacientes con cuerpo lúteo insuficiente, en relación con otros trabajos publicados; esta sería la explicación de la disminución en nuestro trabajo del grupo de pacientes con esterilidad idiopática y el aumento del factor endocrino.

Es necesario llegar al diagnóstico etiológico de la alteración endocrina, con el fin de iniciar un tratamiento específico, obteniéndose una respuesta cada vez mejor.

Se puede apreciar, en relación con el factor tuboperitoneal, la alta frecuencia 
de procesos infecciosos responsables de esta patología, alcanzando hasta un $69 \%$, como resultado del bajo nivel socio-económico y de la desnutrición en que viven; la tuberculosis y las enfermedades venéreas son el flagelo de estas pacientes.

Las intervenciones quirúrgicas en pélvis y en especial la resección cuneiforme de ovario, son responsables en segundo lugar de la esterilidad tuboperitoneal, esto ha traído como consecuen. cia que nosotros no utilicemos esta técnica, máxime que síndrome de ovario androgénico es una entidad endocrina de tratamiento médico y su respuesta, en nuestras manos ha sido buena.

El factor masculino ocupó el tercer lugar con un $26.2 \%$, por lo cual se ve la importancia de programar un estudio conjunto de la pareja con el fin de detectar las causas de esterilidad, y en el caso de encontrarse una patología masculina luego de un estudio adecuado, debe programarse para un tratamientc conjunto con el andrólogo, evitando el empleo de tratamientos empíricos que tanto daño hacen al paciente.

En el supuesto caso de no ser posible el embarazo por alteración irreversible del esposo, se debe aconsejar una adopción o una inseminación artificial, luego de un estudio sicológico adecuado.

Una mala evaluación de la pareja trae sorpresas desagrables en el futuro y resultados funestos; es por ello que el médico debe integrar todos sus conocimientos para tomar la conducta más adecuada, pues de ella depende la felicidad de la pareja.

\section{Conclusiones}

1. Hay necesidad de crear grupos de Ginecología Endocrina y Reproducción Humana (eliminando la palabra esterilidad) en las universidades e instituciones médicas, con el fin de prestar un servicio adecuado, racional y serio a aquellos pacientes que lo solicitan.

2. Se debe establecer y evaluar una metodología para la investigación y tratamiento de las parejas.

3. Debe hacerse un estudio integral de la pareja.

4. Un estudio de la pareja estéril exige la asociación con grupos multidisciplinarios: patólogos, neurólogos, andrólogos, endocrinólogos, siquiatras, etc.

5. Siendo la esterilidad endocrina de alta frecuencia en nuestro medio, es necesario intensificar la enseñanza de esta disciplina.

6. A pesar del progreso en las determinaciones hormonales creemos que es básico su correlación con el estudio de los efectores: citología, moco, temperatura basal, endometrio.

7. La laparoscopia es un procedimiento primario para el estudio del factor tuboperitoneal, en pacientes seleccionados.

8. Se le debe dar un impulso a las técnicas de microcirugía tubárica por la alta frecuencia de factor tuboperitoneal que hay en nuestro medio.

\section{Study of the sterile couple Conclusions and recommendations}

1. It is necessary to create Endocrine Ginecology and Human Reproduc. tion Groups (eliminating the word sterility) at the universities and medical institutions in order to give an appropriate, rational and serious service to those patients requiring it.

2. It is necessary to establish and assess a methodology for the investigation and treatment of the couples.

3. An integral study of the couple should be carried out. 
4. The study of the sterile couple must be linked to multidisciplinary groups: pathologists, neurologists, andrologists, endocrinologists, psychiatrists, etc.

5. Since endocrine sterility is highly frequent in our environment, it is necessary to intensify the teaching of this subject.

6. Notwithstanding the progress in hormonal determinations, we believe it necessary to co-relate it with the effectors' stury: cytology, mucus, basal temperature, endometrium.

7. Laparoscopy is a primary procedure in the study of the tubo-peritoneal factor in selected patients.

8. Tubal microsurgery techniques must be stimulated due to the high frequency of tubo-peritoneal factor in our media.

\section{Bibliografía}

1. NOYES RW., Herting AT, Rock J.: Dating the endometrial biopsy. Fertil Steril 1: 3 , 1950.

2. JONES GES, Madrigal - CV - Hormonal findings in association With anormal corpus luteum function in the Human: The luteal phase defect. Fert Steril 21: 1, 1970.

3. AKSEL S., JONES GES: Effect of Progestetore and $\mathbf{1 7}$ hydroxy progesterone caproate on normal corpus luteum function Am J. Obstet Gynecol 118: 466, 1974.
4. TURNER V., DAVIS CD, CARTER B., DURHAM NC: Correlation of estimated prognosis With some findings and results in $\mathbf{7 5 0}$ sterile couples. Am J. Obstet Gynec 70: 1189, 1955.

5. STONE A. WARD ME: Factors Responsible for pregnancy in $\mathbf{5 0 0}$ infertility cases. Fertil Steril 7: 1, 1956.

6. JONES GES, Pourmand K.: An evalution of etiologia factors and therapy in $\mathbf{5 5 5}$ private patienst with primary infertility. Fertil Steril 13: 398, 1962.

7. DOR J., HOMBURG R., RABAU E.: An evaluation of etiologic Factors and Therapy in 665 infertile couples. Fertil Steril 28: 718 , 1977.

8. DELGADO, U. J., VILLALOBOS, R.M., Wulfovich, B. M. MORALES, F. G. ZERTUICHE, M. R.: Estudio de la pareja estéril, análisis de 500 casos. Ginec Obstet Mex 41: 29, 1977.

9. GARCIA, M. A., SALDANA, G. R., RINCON, C. R. ORDOZA, C. G., GONZALEZ, B. M.: Análisis General de $\mathbf{5 0 0}$ parejas con esterilidad o infertilidad Ginecol. Obstet Mexic. 41: 409, 1977.

10. DIAZ, I. A., CASTILLO Del, V. F. J., DIAZ, I. A. Etiología y tratamiento en 204 parejas estériles. Ginec Obst Mex 45-207, 1979.

11. RIOS De los J.: Valores normales en 500 espermogramas practicados en Profamilia Medellín (no publicado).

12. KISTNER, RW. Endometriosis e infecundidad Clin. Obstet. Ginecol 1: 101, 1979.

13. PERALtA ABELlO M. T., REVOlLO PARDO F., GARCIA CONTI F. Estudio de la pareja estéril en el Hospital San Ignacio. Rev. Col. Obst.-Gin. 30: 122, 1979. 